\title{
MAGNETIC VORTICES IN A GAUGED O(3) SIGMA MODEL WITH SYMMETRY BREAKING SELF-INTERACTION
}

\author{
P.Mukherjee円 \\ Department of Physics \\ A.B.N.Seal College,Coochbehar \\ West Bengal,India
}

\begin{abstract}
We consider a $(2+1)$ dimensional nonlinear $\mathrm{O}(3)$ sigma model with its $\mathrm{U}(1)$ subgroup gauged along with the inclusion of a self-interaction having symmetry breaking minima.The gauge field dynamics is governed by the Maxwell term.The model is shown to support topologically stable purely magnetic self-dual vortices.
\end{abstract}

\footnotetext{
${ }^{1}$ e-mail:subinit@anp.saha.ernet.in
} 
The $2+1$ dimensional $\mathrm{O}(3)$ nonlinear sigma model has been popular over a long period of time[1] due to its own mathematical interest of providing topologically stable soliton solutions which are exactly integrable in the Bogomol'nyi limit [2] and also for its applications in condensed matter physics [3,4]. The topological solitons of the model are classified according to the second homotopy of the field as a mapping from two dimensional space to the internal field space. The solutions are scale invariant which poses a problem for their particle interpretation on quantisation.Numerical computation of solitonic interaction behaviour in the model reveals the problem clearly[5].

A particularly remarkable way of breaking the scale invariance of the model is to partially gauge the global symmetry in the internal space. The gauge field dynamics was initially taken to be governed by the Maxwell term[6].A particular form of self-interaction had to be included to satisfy self-duality conditions. This new self-duality was later extended to models with the Chern-Simons gauge coupling[7]. The topological stability of the solitons of these models owes again to the second homotopy of mappings from the physical to the internal space as in the usual sigma models. These solutions also share a common feature- they are infinitely degenerate in each topological sector.Recently it has been shown in connection with [7] that the degeneracy of the topological solitons of the model is lifted by chosing a self-interaction with symmetry breaking minima [8].The phenomenon of the lifting of the degeneracy was associated with the novel topology introduced by the chosen self-interaction. The topological solitons are now classified according to the first homotopy group. The question comes what happens when this new topology is introduced in the model with the Maxwell term.In the present letter we address this question.

We consider here a $(2+1)$ dimensional nonlinear sigma model with its $U(1)$ subgroup gauged by the Maxwell term.Unlike [6] the posited self- interaction possesses symmetry breaking minima.The topological solitons of the model are electrically neutral carrying magnetic flux which is quantised in each topological sector. The Bogomol'nyi bounds are saturated.We thus observe purely magnetic self dual topological vortices. This is in contrast with [6] where the topological solitons carry arbitrary magnetic flux and thus do not qualify as vortices.Purely magnetic vortices occured in a nonlinear $\mathrm{O}(3)$ sigma model with non-abelian Chern-Simons term[9] and also in an abelian model with non-minimal coupling [10]. In the latter case the solitons are non-topological in nature. Thus it appears that the present model reveals the first occurence of topologically stable purely magnetic vortices in the context of the $\mathrm{O}(3)$ nonlinear sigma model with abelian gauge coupling..

The Lagrangian of our model is given by

$$
\mathcal{L}=\frac{1}{2} D_{\mu} \phi \cdot D^{\mu} \phi-\frac{1}{4} F_{\mu \nu} F^{\mu \nu}+U(\phi)
$$


Here $\phi$ is a triplet of scalar fields constituting a vector in the internal space with unit norm

$$
\begin{array}{r}
\phi_{a}=\mathbf{n}_{\mathbf{a}} \cdot \phi,(a=1,2,3) \\
\phi \cdot \phi=\phi_{a} \phi_{a}=1
\end{array}
$$

where $\mathbf{n}_{a}$ constitute a basis of unit orthogonal vectors in the internal space. We work in the Minkowskian space - time with the metric tensor diagonal, $g_{\mu \nu}=(1,-1,-1)$.

$D_{\mu} \phi$ is the covariant derivative given by

$$
D_{\mu} \phi=\partial_{\mu} \phi+A_{\mu} \mathbf{n}_{3} \times \phi
$$

The $\mathrm{SO}(2)(\mathrm{U}(1))$ subgroup is gauged by the vector potential $A_{\mu}$ whose dynamics is dictated by the Maxwell term. The electromagnetic field tensor

$$
F_{\mu \nu}=\partial_{\mu} A_{\nu}-\partial_{\nu} A_{\mu}
$$

The potential

$$
U(\phi)=-\frac{1}{2} \phi_{3}^{2}
$$

gives our chosen form of self interaction of the fields $\phi_{a}$. Note that the minima of the potential arise when ,

$$
\phi_{3}=0 \text { and } \phi_{1}^{2}+\phi_{2}^{2}=1
$$

Clearly (7) corresponds to the spontaneous breaking of the $\mathrm{U}(1)$ symmetry of (1).

The Euler - Lagrange equations of the system (1) is derived subject to the constraint (3) by the Lagrange multiplier technique

$$
\begin{aligned}
D_{\nu}\left(D^{\nu} \phi\right) & =\left[D_{\nu}\left(D^{\nu} \phi\right) \cdot \phi\right] \phi-\mathbf{n}_{3} \phi_{3}+\phi_{3}^{2} \phi \\
\partial_{\nu} F^{\nu \mu} & =j^{\mu}
\end{aligned}
$$

where

$$
j^{\mu}=-\mathbf{n}_{3} \cdot \mathbf{J}^{\mu} \text { and } \mathbf{J}^{\mu}=\phi \times D^{\mu} \phi
$$

Using (8) we get

$$
D_{\mu} \mathbf{J}^{\mu}=\left(\mathbf{n}_{3} \times \phi\right) \phi_{3}
$$

From (9) we find,for static configurations

$$
\nabla^{2} A^{0}=-A^{0}\left(1-\phi_{3}^{2}\right)
$$


From the last equation it is evident that we can chose

$$
A^{0}=0
$$

As a consequence we find that the excitations of the model are electrically neutral.

By inspection we can construct a conserved current

$$
K_{\mu}=\frac{1}{8 \pi} \epsilon_{\mu \nu \lambda}\left[\phi \cdot D^{\nu} \phi \times D^{\lambda} \phi-F^{\nu \lambda} \phi_{3}\right]
$$

It can be shown easily that

$$
\partial_{\mu} K^{\mu}=0
$$

The corresponding conserved charge is

$$
T=\int d^{2} x K_{0}
$$

Using (14) and (16) we can write

$$
T=\int d^{2} x\left[\frac{1}{8 \pi} \epsilon_{i j} \phi \cdot\left(\partial^{i} \phi \times \partial^{j} \phi\right)\right]+\frac{1}{4 \pi} \int_{\text {boundary }} \phi_{3} A_{\theta} r d \theta
$$

where $\mathrm{r}, \theta$ are polar coordinates in the physical space and $A_{\theta}=\mathbf{e}_{\theta} \cdot \mathbf{A}$. We identify $\mathrm{T}$ with the topological charge of the model.The form (17) will be useful in the following analysis.

We now construct the energy functional from Schwinger's energy - momentum tensor [11] which in the static limit becomes

$$
E=\frac{1}{2} \int d^{2} x\left[\left(D_{i} \phi\right) \cdot\left(D_{i} \phi\right)+F_{12}^{2}+\phi_{3}^{2}\right]
$$

The energy functional (18) is subject to the constraint (3). For finiteness of energy we require $\phi$ to go to one of the symmetry breaking minima given by (7) for points at infinity.The physical infinite circle is thus mapped on the equatorial circle of the internal sphere. This is the new topology we have talked of earlier.Note that for the usual sigma model the topological solutions are characterised by the second homotopy of the field as a mapping from two dimensional space to the internal field space.Distinctively,here the topologically stable solutions are classified according to the homotopy

$$
\Pi_{1}\left(S_{1}\right)=Z
$$

Let us note in passing the influence of the new topology on the admissible values of the topological charge from (17).Wwe find that the second term on te r.h.s. vanishes in 
the limit $\phi_{3} \rightarrow 0$ on the boundary. The first term of the r.h.s. is known to give the number of times the physical space wraps the internal sphere. Clearly, when the equatorial circle is traversed once, the physical space is mapped on a hemisphere of the internal sphere.Thus the topological charge will be given by

$$
T=\frac{n}{2}
$$

where $\mathrm{n}$ is the number of times the equatorial circle is traversed. Evidently half integral values of $\mathrm{T}$ are allowed.

Let us now define

$$
\psi=\phi_{1}+i \phi_{2}
$$

Using the definition of $\psi$ we can write

$$
D_{i} \phi \cdot D_{i} \phi=\left|\left(\partial_{i}+i A_{i}\right) \psi\right|^{2}+\left(\partial_{i} \phi_{3}\right)^{2}
$$

From the discussions of the preceding paragraph we get

$$
\psi \approx e^{i n \theta}
$$

on the physical boundary where $n$ has been defined in (20). From (18) and (22) we observe that for finite energy configurations we require

$$
\mathbf{A}=\mathbf{e}_{\theta} \frac{n}{r}
$$

on the boundary.

The asymptotic form (22) allows us to compute the magnetic flux

$$
\Phi=\int B d^{2} x=\int_{\text {boundary }} A_{\theta} r d \theta=2 \pi n
$$

The above equation shows that the magnetic flux is quantised in each topological sector.Thus in contrast with [6] the topologically stable solutions solutions of the model (1) have quantised magnetic flux. Note that the quantisation of the magnetic flux is ensured by the novel topology (19) the origin of which traces back to the choice of the self-interaction potential (6) having symmetry breaking minima.

Our next persuit is to show that in the present model self-duality conditions can be obtained by satisfying Bogomol'nyi limit.For this purpose we rearrange the energy functional as

$$
E=\frac{1}{2} \int d^{2} x\left[\frac{1}{2}\left(D_{i} \phi \pm \epsilon_{i j} \phi \times D_{j} \phi\right)^{2}+\left(F^{12} \pm \phi_{3}\right)^{2}\right] \pm 4 \pi T
$$


Equation (26) gives the Bogomol'nyi conditions

$$
\begin{aligned}
D_{i} \phi \pm \epsilon_{i j} \phi \times D_{j} \phi & =0 \\
F_{12} \pm \phi_{3} & =0
\end{aligned}
$$

which minimise the energy functional in a particular topological sector, the upper sign corresponds to + ve and the lower sign corresponds to -ve value of the topological charge.The equations can be handled in the usual method[3,6] to show that the scale invariance is removed by the artifice of the gauge-field coupling.

We will now show the consistency of (27) and (28) using the well-known Ansatz[6, 12]

$$
\begin{array}{r}
\phi_{1}(r, \theta)=\sin F(r) \cos n \theta \\
\phi_{2}(r, \theta)=\sin F(r) \sin n \theta \\
\phi_{3}(r, \theta)=\cos F(r) \\
\mathbf{A}(r, \theta)=-\mathbf{e}_{\theta} \frac{n a(r)}{r}
\end{array}
$$

From (7) we observe that we require the boundary condition

$$
F(r) \rightarrow \pm \frac{\pi}{2} \text { as } r \rightarrow \infty
$$

and equation (24) dictates that

$$
a(r) \rightarrow-1 \text { as } r \rightarrow \infty
$$

Remember that equation (24) was obtained so as the solutions have finite energy. Again for the fields to be well defined at the origin we require

$$
F(r) \rightarrow 0 \text { or } \pi \text { and } a(r) \rightarrow 0 \text { as } r \rightarrow 0
$$

Substituting the Ansatz(29) into (27) and (28) we find that

$$
\begin{array}{r}
F^{\prime}(r)= \pm \frac{n(a+1)}{r} \sin F \\
a^{\prime}(r)=\mp \frac{r}{n} \cos F
\end{array}
$$

where the upper sign holds for +ve $\mathrm{T}$ and the lower sign corresponds to -ve T.Equations (33) and (34) are not exactly integrable. They may be solved numerically subject to the appropriate boundary conditions to get the exact profiles. 
Using the Ansatz (29) we can explicitly compute the topological charge T by performing the integration in (17). The result is

$$
T=-\frac{n}{2}[\cos f(\infty)-\cos f(0)]
$$

So we find that according to (30) and (32) $\mathrm{T}= \pm \frac{n}{2}$ which is in agreement with our observation (20). Note that $\mathrm{f}(0)=0$ corresponds to + ve $\mathrm{T}$ and $\mathrm{f}(0)=\pi$ corresponds to -ve $\mathrm{T}$. If we take +ve $\mathrm{T}$ we find $\mathrm{F}(\mathrm{r})$ bounded between 0 and $\frac{\pi}{2}$ is consistent with $(30),(32)$ and (33).Again a(r) bounded between 0 and -1 is consistent with (31),(32) and (34). Thus for + ve topological charge the ansatz (29) with the following boundary conditions

$$
\begin{array}{r}
F(0)=0 \quad a(0)=0 \\
F(\infty)=\frac{\pi}{2} a(\infty)=-1
\end{array}
$$

is consistent with the Bogomol'nyi conditions. Similarly the consistency may be verified for -ve $\mathrm{T}$.

The conclusions are as follows.By chosing a self-interaction with symmetry breaking minima in a nonlinear $\mathrm{O}(3)$ sigma model partially gauged with the Maxwell gauge fields we get neutral purely magnetic vortices. These vortices are topologically stable having quantised magnetic flux in each topological sector.This feature is in clear contrast with the results of the earlier model [6] where the magnetic flux remained arbitrary. We have ascribed the reason of this difference to the novelty of the topology introduced in the model by the posited self-interaction. We have also explicitly demonstrated that the model satisfies Bogomol'nyi limits giving self- dual vortices. These vortices share the desirable feature of the breaking of scale-invariance and are thus suitable for particle interpretation. The consistency of the Bogomol'nyi conditions have been discussed subject to the appropriate boundary conditions. 


\section{References}

[1] A.A. Belavin and A.M. Polyakov,JETP Lett.22(1975) 245.

[2] E.B. Bogomol'nyi,Sov. J. Nucl. Phys.24(1976)449.

[3] R.Rajaraman, Solitons and Instantons (North Holland Publishing Company).

[4] W.J.Zakrzewski,Low dimensional sigma models (A.Hilger,Bristol,1989).

[5] R.A.Leese,M.Peyrard and W.J.Zakrzewski, Nonlinearity 3(1990)387.

[6] B.J.Schroers,Phys.Lett.B 356(1995)291.

[7] P.K.Ghosh and S.K.Ghosh,Phys.Lett.B366(1996)199.

[8] P.Mukherjee,On the question of degeneracy of topological solitons in a gauged $\mathrm{O}(3)$ nonlinear sigma model with Chern-Simons term (to be published in Phys.Lett.B).

[9] G.Nardelli,Phys.Rev.Lett.73(1994)2524.

[10] P.K.Ghosh,Phys.Lett.B 381(1996)237.

[11] J.Schwinger,Phys.Rev.127(1962)324.

[12] Y.S.Yu and A.Zee,Phys.Lett. B147(1984) 325. 